\title{
VIRTUAL DENTAL PATIENT: A SYSTEM FOR VIRTUAL TEETH DRILLING
}

\author{
I. Marras ${ }^{\dagger}$, L. Papaleontiou ${ }^{\dagger}$, N. Nikolaidis ${ }^{\dagger}$, K. Lyroudia ${ }^{\ddagger}$ and I. Pitas ${ }^{\dagger}$ \\ ${ }^{\dagger}$ Department of Informatics \\ Aristotle University of Thessaloniki \\ 54124, Thessaloniki, Greece \\ ${ }^{\ddagger}$ Department of Endodontology, Dental School, \\ Aristotle University of Thessaloniki \\ 54124, Thessaloniki, Greece
}

\begin{abstract}
This paper introduces, a virtual teeth drilling system named Virtual Dental Patient designed to aid dentists in getting acquainted with the teeth anatomy, the handling of drilling instruments and the challenges associated with the drilling procedure. The basic aim of the system is to be used for the training of dental students. The application features a 3D model of the face and the oral cavity that can be adapted to the characteristics of a specific person and animated. Drilling using a haptic device is performed on realistic teeth models (constructed from real data), within the oral cavity. Results and intermediate steps of the drilling procedure can be saved for future use.
\end{abstract}

\section{INTRODUCTION}

Practicing dental drilling for cavity preparation and other similar tasks is an important part of a dental student training. Usually students use artificial teeth and jaws (sometimes placed within a manikin head) and real instruments before proceeding to real patients. Lately, virtual-reality based cavity preparation training systems have been introduced. The DentSim ${ }^{\mathrm{TM}}$ system (Denx Corp, Jerusalem, Israel) [1] comprises of a real dental unit, a manikin head, a tracking system and software that allows student to view the results of his/her cavity preparation in the manikin head on 3D models on a computer monitor and compare them with the results of an optimal preparation. The systems presented in [2], [3] involve a 3D virtual tooth and a Phantom haptic device (Sensable Technologies Inc., Woburn, MA) that enables the trainee to perform virtual drilling while receiving appropriate force feedback. The systems described in both these papers operate on standalone teeth and the focus is on providing realistic material removal simulation as well as realistic, haptically stable and computationally efficient force rendering. However such a simulation would be much more realistic if cavity preparation was performed on teeth placed within the oral cavity.

The Virtual Dental Patient simulator described in this paper allows the user to view/manipulate a 3-D head and oral cavity model constructed using anatomical data, adapt the model to the characteristics of specific patient using either facial photographs or 3D data, animate it using an MPEG-compatible facial animation player and perform virtual tooth drilling within the oral cavity using a Phantom haptic device to control the drilling tool. Drilling is performed on 3D volumetric/surface models of teeth, obtained from cross sections of real teeth. Final results, i.e. teeth models generated by the drilling procedure, as well as intermediate steps of the drilling procedure can be saved for future use. Apart from being used as a training tool for students, the system can also assist experienced dentists in planning a real tooth drilling intervention by getting familiar with the individual patient anatomy, identifying landmarks, planning the approach and deciding upon the ideal target position of the actual drilling activity.

This paper is structured as follows. The construction of the head/oral cavity model is described in Section 2. Model adaptation/personification and animation is described in Sections 3, 4, whereas virtual tooth drilling is presented in Section 5. Conclusions follow.

\section{ANATOMICAL HEAD/ORAL CAVITY MODELING}

In order to achieve realism, a head/oral cavity model comprised of anatomically meaningful 3D points taken on the various head tissues was constructed by using manual modeling techniques at the cost of increased modeling time [4]. We have created such a 3D surface model consisting of 1392 3D points and 2209 triangles distributed among 11 different entities (nodes) (Figure 1) using the publicly available anatomical (cryosections) and CT data of a male cadaver originated from the Visible Human Project (National Institute of Health (NIH), USA) [5]. The model includes the external face area and gums, palate, teeth, tongue, lips, cheeks, larynx and uvula, represented as triangular meshes. For modeling the face, we have used the CANDIDE - 3 [6] face model and adapted it to the NIH data. Essentially, the constructed model provides an MPEG4 compliant extension of the CANDIDE-3 towards both the oral cavity's internal tissue surfaces (which are important for dental applications) and the head - neck outer surface. 

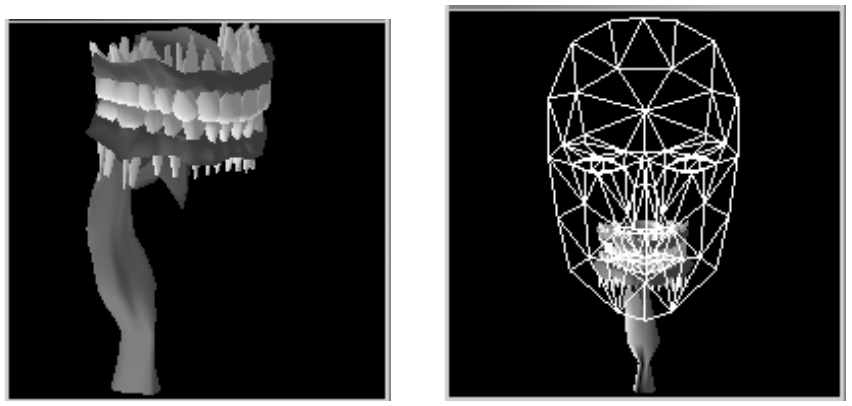

Figure 1: The oral/head cavity 3D model (left and right respectively) utilized in the Virtual Dental Patient application.

\section{MODEL ADAPTATION - PERSONIFICATION}

\subsection{Facial model - oral cavity adaptation}

Within the developed simulator, the user can adapt the generic facial mesh model to the characteristics of a specific person, using a semi-automatic approach that relies on two photographs of the person taken from two perpendicular directions, i.e. a frontal one and a side one (Figure 2). The adaptation is supported by a 2D FEM (Finite Element Model). This approach has been selected because it offers speed and reliability in the adaptation procedure and requires small interaction with the user. The adaptation process comprises of the two steps explained below.

In the first step, the user performs global translation and scaling on the facial model (being overlaid on the two photographs). The models of the internal structures (i.e. those in the oral cavity) are scaled and translated automatically in the same way as the facial model.

In order to proceed in a detailed adaptation of the mesh on the photographs, user interaction along with a 2D FEM [7] method executed in real time is used in the second step. Essentially the model's mesh is represented as a system of springs that can be deformed through user interaction. The adaptation is implemented by having the user moving (by using the mouse) a certain number vertices of the model's mesh, to the corresponding points in the picture. Subsequently, the FEM moves the remaining vertices of the mesh (i.e. those that have not been displaced by the user) move too. This operation is executed in real time.

The FEM-based user-guided adaptation is applied only on the facial model. During this adaptation, the models of the oral cavity structures are translated and scaled in real time according to the position of the model's mouth. More concretely, after each deformation of the facial mesh, the translation of the centre of the mouth (evaluated using FDPs 8.3 and 8.4) is calculated and the models of the internal structures are translated by the same vector. Moreover, the oral cavity models are scaled on the basis of the jaw width which in turn is evaluated using the centers of the cheeks (FDP 5.1 and 5.2).

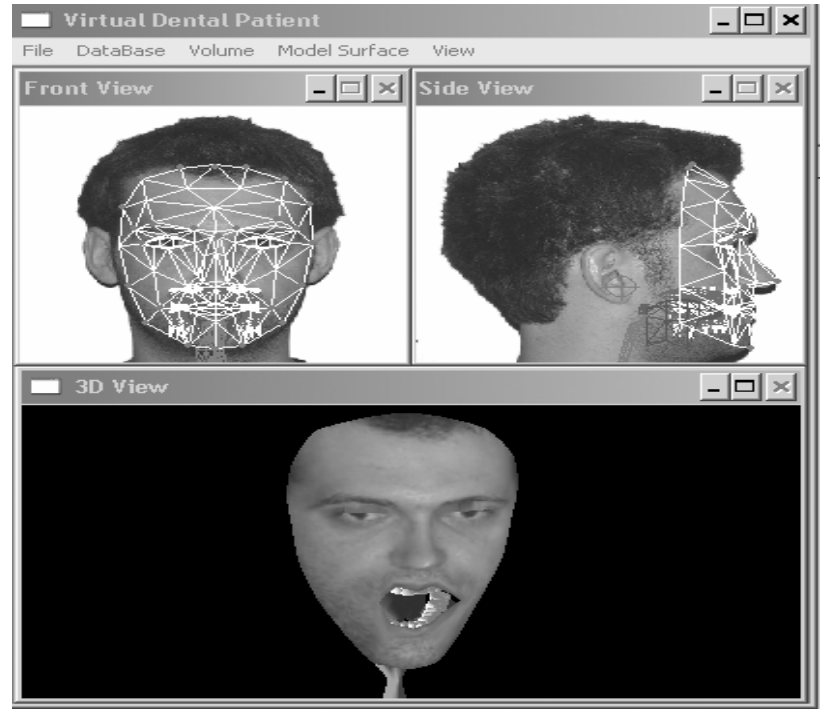

Figure 2: Model adaptation using two facial photographs.

\subsection{Other adaptation procedures}

As a final stage of the model adaptation/personification the user can select through a graphic representation of the teeth the ones that he wishes to remove from the model. This is useful in order to create models of persons that lack some of their teeth. The user can also control which parts of the model (e.g face, gums, tongue) will be visible.

In addition, the user can replace at the 3D Oral Cavity model the limited resolution prototype of a certain tooth where virtual drilling will be performed with a more detailed model. For this operation, the user provides the volumetric model of the tooth in the form of a series of slice images where the structures of interest (external tooth surface and root canals) have been segmented. A database of such volumetric models for all types of teeth (canines, molars, premolars etc) is provided along with the application. This database has been constructed by digitizing and post-processing (alignment and segmentation) physical cross sections of extracted teeth, viewed through an optical microscope.

The application loads the volumetric representation of the tooth (required for the drilling procedure as will be explained later) and also applies the Discrete Marching Cubes algorithm [8] to obtain the surface representation of the tooth and embed it in the face/oral cavity model. Several options of the surface extraction procedure (smoothing of the surface by applying the so-called Laplacian smoothing, merging of all coplanar triangles that will result from the triangulation into larger polygons, etc) can be controlled through an appropriate interface. The selected tooth is inserted in the face/oral cavity model in two steps: 
- Coarse automatic placement of the tooth on the gums by using information about the size of the teeth models and the alveole.

- Optional manual adjustment (global rotation, translation scaling).

After the adaptation, texture mapping can be applied on the $3 \mathrm{D}$ model of the face, for achieving additional realism.

\section{MODEL ANIMATION}

For animating the models, compatibility with the MPEG-4 is provided. More specifically, the application includes a FAP player that reads FAP files from disk and animates the model by moving the corresponding MPEG-4 FDPs. The user can save the model configuration at a certain frame of the animation in VRML format.

The movements of the internal structures (which are not covered by the MPEG model) should be also defined. More specifically, the movement of the lower jaw which is a rotation around an axis should be defined. The user specifies this axis during the model adaptation procedure described above and the movement of the vertex that represents the chin, i.e., FDP 2.1 is used to define the angle of rotation of the jaw around this axis. The lower teeth, gum and tongue are also rotated along with the jaw. Furthermore the lower jaw can move horizontally. In order to implement this movement, the influenced sub-models (teeth, gums, jaw, etc) are translated in the $\mathrm{x}$ axis at the same distance and direction as the vertex that defines the tip of the chin (FDP 2.1).

During the animation, real-time surface subdivision is used in order to improve the quality of the models and obtain smooth surfaces. A technique that leaves the original model vertices unaffected is needed in this case. After experimentation, the curved Point-Normal triangles (curved PN triangles) technique [9] has been selected.

\section{VIRTUAL TOOTH DRILLING}

When done with loading a detailed tooth model on the application the user can proceed in performing virtual drilling on this tooth. When loading such a model both its volumetric and surface representation are available to the application. The reason for this is that in the proposed approach, removal of material during drilling is implemented as a series of morphological operations on the volumetric (voxel-based) representation of the object. More specifically, in the virtual drilling approach that has been developed, drilling operations are simulated using successive erosions involving 3-D structuring elements, which represent the shape of drilling tools (dental burs) [10]. This approach can be used to implement almost any kind of drilling tool shape. Four basic tool shapes, a spherical, a cylindrical, a cylindrical-conical and a conical (which is a special case of the cylindrical-conical tool) have been implemented using 3-D mathematical morphology decomposition. The user can select the appropriate drilling tool and control its shape parameters (e.g. the radius for the spherical tool). The parameters of a tool can be saved for future use. Moreover, a database of virtual dental burs with dimensions taken from real burs has been created and accompanies the application.

At this stage the user can perform on the volumetric tooth model two additional operations:

1) Add material on the surface of the volume. This is implemented by using 3D dilations.

2) Smooth the surface of the volume locally, by using the mouse and by selecting an appropriate smoothing window.

The dental drilling operation can be performed directly on the standalone volumetric model of the tooth using the mouse. However, more realism can be achieved by performing drilling on the surface model of the inserted tooth, within the full face-oral cavity model, using either the mouse or a haptic device to control the operation (Figure 3). The Phantom Desktop six degrees of freedom positional sensing force-feedback haptic device has been used for this purpose.

Using the haptic device, the user can proceed in drilling, sensing contact/resistance forces. Currently, a constant force model is utilized. The force value is determined by the properties (stiffness, static and dynamic friction) of the object, as set by the user. The stylus of the haptic device controls the position of the dental bur [11] (which is also visible as a simple surface model on the scene). During the material removal operation, the application makes use of the internal dual surface/volumetric representation of the tooth. This dual representation is necessary since the GHOSTTM SDK (Sensable Technologies Inc. Woburn, MA) that is used for handling the tool-tooth physical interaction (e.g. collision detection) requires that the object is represented by its surface whereas the proposed mathematical morphologybased material removal algorithm operates on the volumetric representation of the tooth. In other words, the position of the tip of the drilling tool on the surface representation of the tooth (obtained through collision detection) is used to find the corresponding position on the volumetric representation. Then $3 \mathrm{D}$ erosions with the appropriate structuring element are performed at this position of the volumetric model and the surface model is updated on the haptic device scene graph. In order to reduce the update time, local updating of the surface model is performed by local application of the Discrete Marching Cubes algorithm.

The user can select whether the performed drilling operations on the tooth model will be tracked, i.e., if a "history" of the performed operations will be kept or not. If the user chooses to keep track of these operations he can undo a certain number of them. Furthermore, he can save 
the sequence of operations on a file and re-apply them on the current tooth.

The tooth that has been subject to a drilling operation can be saved either in volumetric representation (i.e. as a series of slices) or as a triangular mesh. The supported mesh formats are VRML2, DXF and STL. An example of a tooth that has been drilled with the developed application can be seen in Figure 4. During drilling, the user can enable stereo viewing through active shutter glasses. In addition, transparency options are provided.

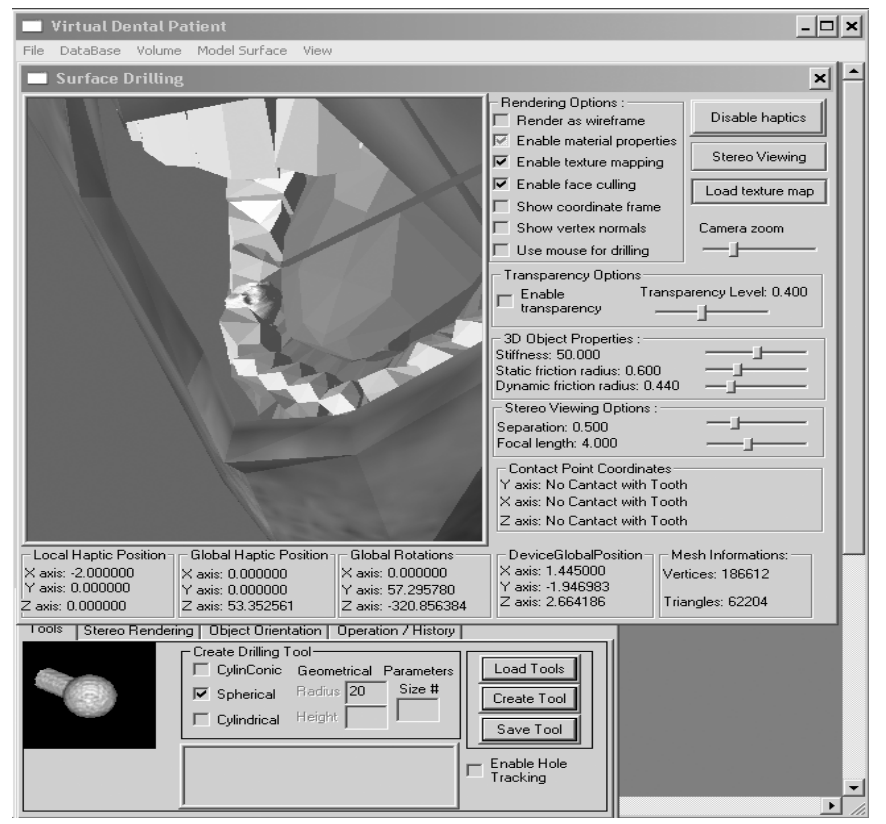

Figure 3: Performing virtual drilling on the face/oral cavity model.
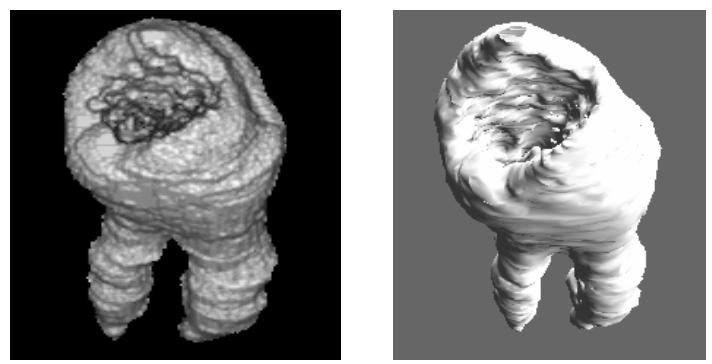

Figure 4: Volumetric (left) and surface (right) representation of a tooth that has been subject to virtual drilling.

\section{CONCLUSIONS-FUTURE WORK}

The Virtual Dental Patient tooth drilling simulator has been described in this paper. The application allows the user to practice virtual tooth drilling for cavity preparation and similar tasks on detailed teeth models placed within an adaptable and animated 3D head and oral cavity model. A Phantom haptic device can be used to provide force feedback during the operation. So far the focus was on developing the "visual" part of the application. Future work will focus on developing realistic and physically accurate material removal and force models that will take into account all the involved factors.

\section{ACKNOWLEDGEMENTS}

This work has been conducted in conjunction with the "SIMILAR" European Network of Excellence on Multimodal Interfaces of the IST Programme of the European Union (www.similar.cc).

\section{REFERENCES}

[1] Rose JT, Buchanan J, Sarrett DC. "Software reviews - the DentSim system”. Journal of Dental Education, Vol. 63, 1999, pp. 421-423.

[2] J.F. Ranta and W.A. Aviles, “The Virtual Reality Dental Training System-Simulating Dental Procedures for the Purpose of Training Dental Students Using Haptics," Proc. Fourth PHANTOM Users Group Workshop, Nov. 1999.

[3] Wang, D., Yuru Zhang, Yuhui Wang, Lee, Y.-S., Peijun Lu, Yong Wang, "Cutting on triangle mesh: local model-based haptic display for dental preparation surgery simulation”, IEEE

Transactions on Visualization and Computer Graphics, Volume 11, Issue 6, Nov.-Dec. 2005 Page(s):671-683.

[4] G. Moschos, N. Nikolaidis and I. Pitas, “Anatomically-Based 3D Face and Oral Cavity Model for Creating Virtual Medical Patients”, IEEE ICME 2004, Taipei, Taiwan, June 2004.

[5] R. Banvard, "The Visible Human Project Image Data Set: From Interception to Completion and Beyond”, CODATA 2002.

[6] J. Ahlberg, "Candide-3 - an updated parameterized face”, technical report no. lith-isy-r-2326, 2001.

[7] K.-J. Bathe, "Finite Element Procedures in Engineering Analysis”, Prentice Hall, 1982.

[8] Montani, R. Scateni, and R. Scopigno "Discretized Marching Cubes”, Visualization '94, pages 281-287, 1994.

[9] A. Vlachos, J. Peters, C. Boyd, J. L. Mitchell, «Curved PN Triangles», ACM Symposium on Interactive 3D Graphics, 2001.

[10] N.Nikopoulos and I.Pitas, " An Efficient Algorithm for 3D Binary Morphological Transformations with 3D Structuring Elements of Arbitrary Size and Shape", in Proc. of 1997 IEEE Workshop on Nonlinear Signal and Image Processing (NSIP'97).

[11] Conti F, Barbagli F, Morris D, Sewell C. CHAI: An OpenSource Library for the Rapid Development of Haptic Scenes Demo paper presented at IEEE World Haptics, Pisa, Italy, March 2005. 